\title{
A Research on Emergency DC Power Support Based on DCOI
}

\author{
Fan Fan, Yingmin Zhang, Xingyuan Li, Wei Ma \\ School of Electrical Engineering and Information, Key laboratory of Smart Grid Sichuan University, Chengdu, China \\ Email: fanfanolginate@sina.com
}

Received October, 2012

\begin{abstract}
An integrated scheme of emergency DC power support which is based on dominant center of Inertia DCOI is proposed to improve power system transient stability. The inter-area speed differences equivalent by dominant center of inertia alleviates the main weakness of the traditional signal which is made of the frequencies at both sides and is represent of abnormal information of the nonlinear dynamic behavior of the power system based on a model in which two AC systems are connected by a HVDC Link. Sichuan Power Grid connected with Southwest Power Grid by Deyang-Baoji HVDC project is used to testify the method. The simulation results show that the control strategy can significantly advance the transient stability of AC/DC system through extended equal area criterion (EEAC).
\end{abstract}

Keywords: DCOI; Speed Differences; EEAC

\section{Introduction}

The main appeal of HVDC (High Voltage Direct Current) with long-distance and large capacity competence for power transmission is the prominent ability to regulate reactive and active power. HVDC improves an efficient and robust approach to transient stability by modulating DC power into AC grid rapidly, which will remedy power imbalance between sending-grid and receiving- grid in order to advance transient angle stability and improve last-low-voltage level or voltage vibrating conditions [1].

Emergency control is a most significant measure against large disturbances which are the gravest threat to the stability to transient security of power systems. Thus, it becomes necessary to choose the most useful DC modulation signal from the huge amount of in- formation for the system stability control services [2]. This paper identifies and selects the "dangerous generators" to make the dominant center of inertia while discarding all "harmless" ones by appraising the kinetic energy as the index in the during-fault period respectively. The inter-area speed differences equivalent by dominant center of inertia can effectively reflect the abnormal information of the nonlinear dynamic behavior of the power system based on a model in which two AC systems are connected by a HVDC link. Accordingly it is as the controller input signal [3-6].

DC power lift / drop-back can provide certain transfers of power for the AC system to ensure the stability of the system by modifying the instructions of the DC system power to increase or reduce the DC transmission power. For instance, when there happens a generator loss in the sending-grid system, DC power drop-back can reduce the DC power to balance the system's active power shortfall [7].

In this paper, an emergency DC power support comprehensive strategy is devised by combining DC power lift / drop-back with the dominant center of inertia speed deviation signal to modify the power set value jointly. Taking Sichuan power grid for background, it indicates that the signal deriving from the dominant center of inertia is more efficient than the traditional signal made of the frequencies at both sides after comparative analysis of the system characteristics of various operating conditions under typical faults.

\section{Mechanism of Edcps Comprhensive Strategy}

In the transient process, the separation of the system is not decided by the energy of the whole system. The unstable situation of the system is caused by a small number of out-of-step generators seriously deviating from the system and most of the generators with each other can still maintain synchronous operation. If all seriously disturbed crew in the system is stabilized, the system is stable; Otherwise, the system is unstable. Thus, the stability of the system is converted to determine the stability of the severely disturbed generators. During a failure, transient energy injected into the system is converted into the 
absolute kinetic energy increment of each generator set. Kinetic and potential energy convert into each other during the process of power angle swing $[8,9]$. Under the center of inertia (COI) coordinates each generator rotor equations of motion are shown as follow:

$$
\begin{aligned}
\frac{\mathrm{d} \delta_{i}}{\mathrm{~d} t}=\tilde{\omega}_{i} & \\
M_{i} \frac{\mathrm{d} \tilde{\omega}_{i}}{\mathrm{~d} t}= & P_{\mathrm{m} i}-P_{\mathrm{e} i}-\frac{M_{i}}{M_{T}} P_{\mathrm{COI}}-D_{i}\left(\tilde{\omega}_{i}+\omega_{\mathrm{COI}}\right) \\
& +\frac{M_{i}}{M_{T}} \sum_{i=1}^{n} D_{i}\left(\tilde{\omega}_{i}+\omega_{\mathrm{COI}}\right)
\end{aligned}
$$

where

$M_{T}$ : inertia time constant of system

$M_{i}$ : inertia time constant of generator

$\delta_{I}:$ power angel of generator

$\omega_{i}:$ angular velocity deviation

$P_{m i}:$ input mechanical power

$P_{e i}$ : output electromagnetic power

$P_{\text {COI }}$ : accelerating power of COI

Transient energy of the system is expressed as follow:

$$
\begin{aligned}
V & =\sum_{i=1}^{n} \int_{\delta_{s i}}^{\delta_{i}}\left[M_{i} \tilde{\omega}_{i}-\left(P_{m i}-P_{e i}-\frac{M_{i}}{M_{T}} P_{\mathrm{COI}}\right)\right] \mathrm{d} \delta_{i} \\
& =\frac{1}{2} \sum_{i=1}^{n} M_{i} \tilde{\omega}_{i}^{2}-\sum_{i=1}^{n} \int_{\delta_{s i}}^{\delta_{i}}\left(P_{m i}-P_{e i}-\frac{M_{i}}{M_{T}} P_{\mathrm{COI}}\right) \mathrm{d} \delta_{i} \\
& =V_{k}+V_{p}
\end{aligned}
$$

Take the derivative of $V$ as the change rate of the transient energy:

$$
\begin{aligned}
\frac{\mathrm{d} V}{\mathrm{~d} t}= & \sum_{i=1}^{n} \tilde{\omega}_{i}\left[M_{i} \tilde{\omega}_{i} \frac{\mathrm{d} \tilde{\omega}_{i}}{\mathrm{~d} t}-\left(P_{\mathrm{m} i}-P_{\mathrm{e} i}-\frac{M_{i}}{M_{T}} P_{\mathrm{COI}}\right)\right]= \\
& \sum_{i=1}^{n} \tilde{\omega}_{i}\left[-D_{i}\left(\tilde{\omega}_{i}+\omega_{\mathrm{COI}}\right)+\frac{M_{i}}{M_{T}} \sum_{i=1}^{n} D_{i}\left(\tilde{\omega}_{i}+\omega_{\mathrm{COI}}\right)\right]
\end{aligned}
$$

$V$ Consists of two parts which are the rate of relative kinetic energy into potential energy and the rate of potential energy into relative kinetic energy. If change the rate of the transient energy to be less than zero or fluctuate around zero and gradually decreases over time, the system has a larger stability margin. The kinetic energy of the relative motion between the center of inertia of the critical group and the remaining group can truly reflects out of sync information under dual-machine equivalents mode:

$$
V_{k}=\frac{1}{2} M \omega^{2}
$$

Kinetic energy is expressed by the speed and accordingly the equivalent of two machine speed deviation can be used as a feedback signal to realize emergency control. And the DC power modulation amount generated by the feedback signal is represented as follow:

$$
\Delta P_{\mathrm{d}}=K\left(\omega_{A}-\omega_{B}\right) K>0
$$

Early in the large disturbance, the generators absorbing kinetic energy deviate from the synchronous speed and gradually evolve into the relative movement of the two generator group. The greater the relative kinetic energy of the two generator groups, the greater the likelyhood of system instability [10]. After large disturbance, all generators can be divided into two groups, namely severely disturbed groups (S groups) and remnant groups ( $\mathrm{R}$ groups), and then the whole system can be equaled into dual-machine-unstable model. For the time-varying two-machine system, the NRP (not return point) which decides the stability is the DSP (dynamic saddle point) where the value of image acceleration power is zero. During the process of the first swing, first determine the moment when acceleration kinetic energy of the single machine for critical group reaches a maximum [11,12]. And then selecting the generator maximum acceleration kinetic of this moment as the benchmark, participation factor is defined as the ratio of each generator acceleration kinetic energy with the reference value; For remnant groups, first calculate each generator deceleration kinetic energy the moment disturbed trajectory get through the DSP and take the same critical group generator acceleration kinetic energy maximum value as a reference, therefore the participation factor is defined as the ratio of each generator deceleration kinetic energy with the reference value. To make the formation of the dominant center of inertia (the Dominant Center of Inertia, DCOI), selected generators of which participation factors are greater than 0. 5. According to formula (1), formula (2), formula (12), the control input signal of the emergency power support is integrated by speed deviation information extracted from the two DCOIs.

$$
\begin{aligned}
& \omega_{A}=\frac{\sum_{i \in A} M_{i} \omega_{i}}{M_{A}} \\
& \delta_{A}=\frac{\sum_{i \in A} M_{i} \delta_{i}}{M_{A}} \\
& M_{A}=\sum_{i \in A} M_{i} \\
& \omega_{B}=\frac{\sum_{j \in B} M_{j} \omega_{j}}{M_{B}} \\
& \delta_{B}=\frac{\sum_{j \in B} M_{j} \delta_{j}}{M_{B}} \\
& M_{B}=\sum_{j \in B} M_{j}
\end{aligned}
$$

where: 
$M_{i}$ : inertia time constant of generator in A group

$M_{j}$ : inertia time constant of generator in B group

$M_{A}$ : inertia time constant of the equivalent generator A

$M_{B}$ : inertia time constant of the equivalent generator $\mathrm{B}$

$\delta_{i}: \quad$ power angel of generator in A group

$\delta_{j}: \quad$ power angel of generator in B group

$\delta_{A}: \quad$ power angel of the equivalent generator $\mathrm{A}$

$\delta_{B}: \quad$ power angel of the equivalent generator B

$\omega_{i}:$ angular velocity deviation in A group

$\omega_{j}:$ angular velocity deviation in B group

$\omega_{A}$ : angular velocity deviation of the equivalent generator $\mathrm{A}$

$\omega_{B}$ : angular velocity deviation of the equivalent generator $\mathrm{B}$

\section{Control Effect}

Taking Sichuan power grid in the summer peak load period for background, while Debao HVDC system operating in monopolar mode, this paper focuses on the impact of EDCPS strategies on transient stability in Sichuan power grid. EDCPS strategies are as follows: 1) DC modulation based on the traditional inertia center (TIC); 2) the speed deviations modulation based on the dominant center of inertia (DCOI); 3) the comprehensive EDCPS strategy based on the dominant center of inertia the speed deviations modulation cooperating with DC power upgrade / drop back(CEDCPS).

Consider the following fault: the fault-free outage of generating unit in Ertan Hydropower Station. Investigate the kinetic energy of the generator of the critical group and the remaining groups respectively. Kinetic energy of Ertan unit increases the maximum, which is followed by the order of the Jialing, Jintang factory, Jiangyou, Pubugou. The detailed information is shown in Table 1. It shows that Ertan, Jialing, Panzhihua and Jintang plant units satisfy the conditional and take them to form the dominant center of inertia of group A. Compare the effect of the three emergency power support strategies for improving the system transient stability through the following simulation [13].

Table 1. The largest accelerating power of generator ruing the fault period.

\begin{tabular}{cccc}
\hline Unit name & Acceleration power & Unit name & Acceleration power \\
\hline Ertan & 12.2822 & Tianwan & 1.6665 \\
Jialing & 10.7820 & Ziyili & 1.0577 \\
Panzhihua & 6.6472 & Shiziping & 0.9811 \\
Jintang & 6.2675 & Xiaotianhu & 0.8252 \\
Pubugou & 4.1985 & Tianlonghu & 0.6188 \\
Fuxi & 4.0891 & Weizhou & 0.5188 \\
Baozhou & 3.7990 & Xuecheng & 0.3138 \\
Baozhu & 2.5998 & Chibusu & 0.2657 \\
\hline
\end{tabular}

Figure 1 shows the degree of oscillation of curve 2 is stronger than curve1, which is also consistent with the fact that the kinetic energy of generator units disturbed seriously is lager than the others.

The fault results in not only the serious system oscillation but also the power shortage which is up to $320 \mathrm{MW}$ in Sichuan Power Grid. The power shortage is made up by droping the DC power. The three DC power control strategies all can effectively drop the DC power to enhance the transient stability of the system. Analyzing Table 1, Figure 2 and Figure 3 it can be obviously seen that the improving effect of policy 3 is the best and Strategy 2 is better than Strategy 1 .

Figure 2 tells the trend of the Ertan unit power angle and Figure 3 tells the trend of the Huangyan - Wanxian active power by different control strategies in the postfault.

The oscillation in system was caused by the serious fault during the angle-swing-up course. Strategy 2 taken the DOCI speed deviation signal which can effectively

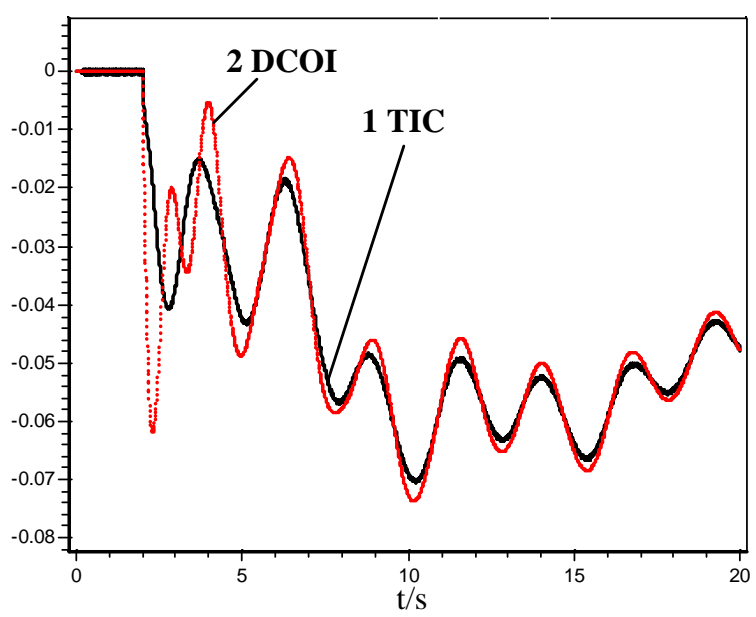

Figure 1. Moudulation SIGNAL Curves(HZ).



Figure 2. Power angle curves of ertan under different modulation signal for EDCPS (Degree). 


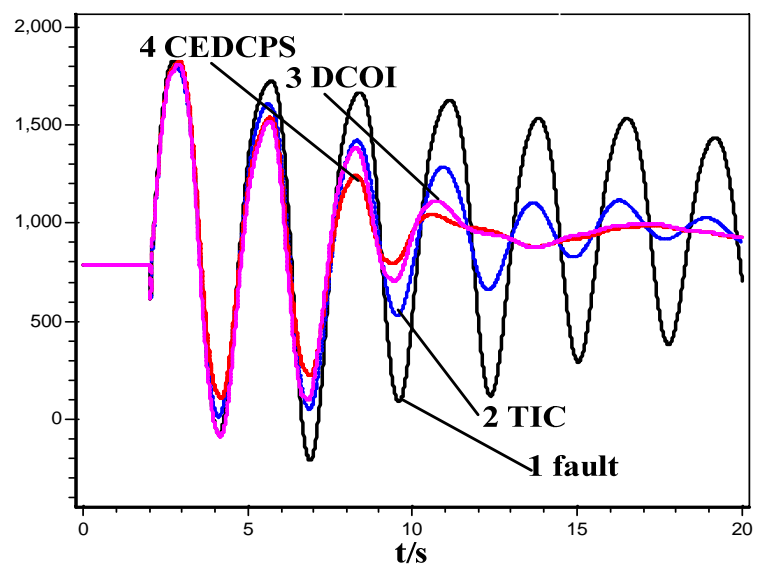

Figure 3. Active power of Huangyan-Wanxian lines (MW).

reflect the inter-area oscillation information provides more damping compared to strategy 1 which is limited by local information. Strategy 3 with the same ability of providing damping as strategy 2 makes up the power shrotage to restore the system to a stable operating point close to the pre-failure stable equilibrium point by droping DC power.

\section{Conclusions}

This paper proposes a wide area information filtering and integration method based on participation factor which is defined by relative kinetic energy increase during the transient process. It provides an effective way for screening and selection of the feedback control signal from the massive information. Compared with traditionas signal modulation, the selected signal with higher accuracy can not only reflect the global features of the system, but also greatly reduce the amount of computation. After a comparative study of the system characteristics of various EDCPS under typical faults, it indicates that the DOCI speed deviation as a control signal can significantly enhance the inter-area damping. The emergency power support comprehensive strategy based on the dominate center of inertia speed deviation modulation cooperating with DC power increase / back drop further develop and utilize the potential of DC modulation to maintain system transient stability. It provides a new way for detection and effective correction of instability threat caused by severe disturbance.

\section{Acknowledgements}

This work is supported by National Natural Science Foundation of China (No. 51037003) and National High Technology Research and Development Program of China (863 Program). At the same time, the authors would like to appreciate all research members of the re- search team for valuable discussion and investigation.

\section{REFERENCES}

[1] X.-Y. Li, "High Voltage Direct Current Transmission System Operation and Control” Beijing: Science Press, 1998.

[2] E. Bristol, "On a New Measure of Interaction for Multivariable Process Control," IEEE Transaction on Automation Control,Vol. 11, No. 1, 1966, pp. 133-134. doi:10.1109/TAC.1966.1098266

[3] P. X. Zhang, Y. J. Cao, H. F. Wang, et al., “Application of Relative Gain Array Method to Analyze Interaction of Multi-Functional FACTS Controllers," Proceedings of the CSEE, 2004, Vol. 24, No. 7, pp. 13-17.

[4] J. Y. Zhang and Y. Z. Sun, "A Vulnerable Cut Sets Based Sensitivity Analysis Method for Locating Emergency Control," Power System Technology, Vol. 31, No. 11, 2007, pp. 1-7.

[5] X. M. Mao, Y. Zhang and L. Guan, "A Novel WAMS Based HVDC Modulation Method," Automation of Electric Power Systems, Vol. 31, No. 7, 2007, pp. 45-49.

[6] J. B. He, L. C. Li, H. X. Chen, et al., "Selection of Feedback Signal for Power System Damping Controller Based on Wide Area Measurements," Automation of Electric Systems, Vol. 31, No. 9, 2007, pp. 6-10.

[7] W. D. Yang, Y. S. Xue, Y. Jing, et al., "Emergency DC Power Support to AC Power System in the South China Power Grid," Automation of Electric Power Systems, Vol. 27, No. 7, 2003, pp. 68-72.

[8] Y. Zhang, L. Wehenkel, P. Rousseaux, et al., "SIME: Ahybrid Approach to Fast Transient Stability Assessment and Contingency Selection,” Electrical Power Energy Syst, 1997, Vol. 19, No. 3, pp. 195-208. doi:10.1016/S0142-0615(96)00047-6

[9] Y. S. Xue, “A Critical Comparison of Various Methods for Transient Stability Assessment-Part Two Barrier Point and the Observation Point," Automation of Electric Power Systems, Vol. 25, No. 11, 2001, pp. 1-7.

[10] M. C. Liao, G. L. Cai and Y. J. Zhang, “Transient Voltage Stability of Received Power Grid in AC/DC Hybrid Power Systems," Power System Protection and Control, Vol. 37, No. 10, 2009, pp. 1-4.

[11] H. J. Wu, Y. H. Wang, X. Y. Li, et al., "Study on DC modulation for AC/DC hybrid transmission system based on comprehensive performance index," Power System Protection and Control, Vol. 38, No. 22, 2010, pp. 68-73.

[12] M. M. Xu, X. Y. Li, Y. H. Wang, et al., "Impact on the Damping Characteristics of Sichuan Power Grid by Debao DC Modulation,” Power System Protection and Control, Vol. 38, No. 23, 2010, pp. 141-146.

[13] T. S. Xu, B. J. Li, Y. H. Bao, et al., "Optimal Parameter-Setting of Under Frequency and Under Voltage Load Shedding for Transient Security," Automation of Electric Power System, Vol. 27, No. 22, 2003, pp.12-15. 